\title{
Evaluación, mapas conceptuales y ego académico en la enseñanza bilingüe en Educación Primaria
}

\author{
Assessment, Concept Mapping and Academic Ego in the Bi- \\ Lingual Primary Education Teaching
}

\section{JOSÉ LUIS GÓMEZ}

Departamento de Pedagogía. Universidad Castilla-La Mancha. (Campus de Albacete). (D) https://orcid.org/0000-0003-3347-0033

\section{JUAN LIRIO}

Departamento de Pedagogía. Universidad Castilla-La Mancha. (Campus de Toledo). (D) https://orcid.org/0000-0002-7467-2233

ISABEL MARÍA GÓMEZ

Departamento de Pedagogía. Universidad Castilla-La Mancha. (Campus de Albacete). (1) https://orcid.org/0000-0002-0964-0923

\section{ROSA MARÍA SERNA}

Departamento de Pedagogía. Universidad Castilla-La Mancha. (Campus de Albacete). (D) https://orcid.org/0000-0003-7537-3582

\section{RESUMEN}

Aunque los programas bilingües de inglés-español llevan tiempo implementándose en España, recientes informes de evaluación realizados en Educación Primaria indican que estos son susceptibles de perfeccionamiento (Dobson, Pérez, y Johnstone, 2010). Con la finalidad de contribuir a su mejora, presentamos una propuesta fundamentada en el empleo de mapas conceptuales para la evaluación formativa y sumativa del aprendizaje integrado de contenidos en lengua extranjera (AICLE) en las asignaturas no instrumentales, también conocidas como non-linguistic disciplines (DNL). Del mismo modo que la evaluación constituye una forma de retroalimentación necesaria para docentes y discentes, el modo en el cual el instructor evalúa el conocimiento del alumnado se vería reflejado en el resultado académico obtenido en tales pruebas de retroalimentación; además de en un mayor o menor tiempo mediante el cual los aprendices recuerdan lo previamente aprendido (Hoffmann, 2003). Consecuentemente, el presente trabajo se presenta como una breve revisión bibliográfica para estimular la reflexión de los docentes DNL en la posible influencia del debilitamiento o reforzamiento del ego académico de los examinandos según el diseño de las pruebas de evaluación.

\section{ABSTRACT}

Although bilingual programmes were implemented in Spain a few years ago, a recent report on these English-Spanish programmes points out that they can still be improved (Dobson, Pérez y Johnstone, 2010). To improve these programmes, we aim to present a proposal based on the use of concept maps for the formative and summative assessment in content and language integrated learning (CLIL) developed in non-linguistic disciplines (DNL). Since evaluation constitutes a method of necessary feedback for teachers and students, the method instructors use to measure students' knowledge will impact the students' school performance. Feedback will also help learners to recall previously learned information. (Hoffmann, 2003). Consequently, this paper is presented as a brief bibliographic review to make DNL teachers reflect on the way summative exam design impacts the students' academic ego during evaluation.

Para citar este artículo: Gómez, J. L., Lirio, J., Gómez, I. M. y Serna, R. M. (2019). Evaluación, mapas conceptuales y ego académico en la enseñanza bi-

Recibido: 09/04/19

Aceptado: 05/09/19

PALABRAS CLAVES Evaluación, bilingüismo, mapas conceptuales, Educación Primaria, aprendizaje, ego.

\section{KEYWORDS}

Evaluation, Bilingualism, Concept Maps, Primary Education, Learning, Ego. 


\section{INTRODUCCIÓN}

En cualquiera de sus variantes la evaluación en educación es imprescindible, pues constituye una forma de retroalimentación esencial para instructores y aprendices. Tanto es así que "mucho de lo que sucede a las personas en sus vidas está basado en la evaluación” (Novak, 2010, p. 14). El mayor o menor éxito en un examen académico, laboral, o cualquier otra de las numerosas pruebas realizadas, poseen gran influencia en el futuro de los examinandos. "Desafortunadamente, gran parte de la 'valoración' realizada es realmente pobre evaluando las competencias humanas" (Novak, 2010, p. 14). Asimismo, en numerosas ocasiones se emplean instrumentos de evaluación, medición y retroalimentación, en cierto modo, inadecuados donde la creatividad y el aprendizaje significativo son comprobados mediante modelos de evaluación diseñados para dar respuesta a un aprendizaje literal y memorístico. Del producto obtenido de una evaluación basada en la memorización, cabe indicar que lo aprendido carecerá de valor para el futuro aprendizaje ya que, aunque permita al aprendiz superar determinadas pruebas de examen, contribuirá escasamente como subsumidor para la adquisición de nuevos conocimientos (Edmondson, 2000) y la posterior transferencia de lo aprendido.

Aunque se han realizado mejoras en el proceso de evaluación, esta necesita de nuevos métodos y enfoques puesto que, cuando se trata de comprobar aprendizaje significativo, algunas pruebas de evaluación presentan enormes deficiencias (Hoffmann, 2003). Esta particular característica se debe a que, generalmente, se ignora que existe una enorme correlación entre la evaluación y el modo en que la instrucción del contenido se desarrolla a lo largo del proceso de enseñanza y aprendizaje (Gipps, 1994). Independientemente de que algunas pruebas sumativas planteen estándares elevados, su diseño, en numerosas ocasiones, se plantea alejado de la evaluación formativa y enfatiza en la habilidad de recordar hechos o unidades aisladas de conocimiento (Black y Wiliam, 1998); este factor contribuiría a que los exámenes constituyan una fuente de estrés para los examinandos (Joshi, Sangahvi, Upadhayaya, Chauhan y Halvadia, 2012). Además del factor de tensión, otro efecto negativo de las pruebas sumativas se referiría a la mayor importancia otorgada por los examinandos al resultado académico, en lugar de al aprendizaje (NUT, 2010).

Del mismo modo que el factor emocional ejerce una influencia en el aprendizaje (Ausubel, Novak y Hanesian, 1983), en la realización de las pruebas sumativas los aprendices igualmente experimentan elevados niveles de ansiedad que influirían en los resultados obtenidos de las mismas. En un estudio realizado por Ng, Koh y Chia (2003) se observó que, previamente a la realización de las pruebas de examen, los individuos que presentaban un mayor nivel de estrés igualmente manifestaban los puntajes más bajos en los resultados académicos obtenidos. En la enseñanza formal, especialmente la vinculada a los programas bilingües en la etapa educativa de Primaria, debería incrementarse el interés por conocer las reacciones que experimentan los aprendices respecto de las pruebas de examen que han de realizar durante esta etapa. La observación y el análisis de estas reacciones contribuiría a la detección de determinadas situaciones y sentimientos como la baja autoestima, el sentimiento de fracaso o el acoso escolar en los sujetos (Shepard y Smith, 1987). Para Claxton (1984) las emociones de los aprendices conforman una parte integral de su aprendizaje, por lo que sentimientos como la frustración y la ansiedad han de ser considerados en la evaluación.

Según Claxton (1984), durante la realización de una prueba de examen, la ansiedad constituye un estímulo positivo cuando la tarea se afronta como un reto que se puede resolver, no como la amenaza, conflicto o problema que no puede ser resuelto. Durante la realización de los exámenes, las percepciones y distinciones entre el reto o amenaza que representan las pruebas conforman factores subjetivos condicionados por la autoestima de los examinandos y la complejidad de las tareas que estos han de realizar. Este factor tendría que hacer reflexionar a los 
educadores, para que tomen conciencia de que "necesitamos enfatizar la evaluación del aspecto sentimiento sobre la experiencia educacional bastante más de lo que actualmente hacemos” (Novak, 2010, p. 194). Aunque pudiese parecer irrelevante, obtener retroalimentación sobre cómo se siente el aprendiz respecto de sus pares, así como qué percepción posee sobre lo que los demás opinan de él posee gran relevancia para docentes y discentes (Novak, 2010). En numerosas ocasiones, la mayor o menor autoestima o ego académico será parte del reflejo de lo que tanto el grupo de aula y el docente espera de cada discente en los resultados de las pruebas de examen; pudiendo determinar las actuaciones del alumnado.

Respecto de la importancia del ego en el contexto educativo, podríamos resaltar el hecho de que "maestros que no tienen una fuerte percepción del ego de 'estoy bien' [y que] frecuentemente bajo una imperceptible o explícita forma atacan el ego de sus estudiantes" (Novak, 2010, p. 157) influyendo en la predisposición de estos últimos por aprender. Por esta razón es importante que el docente construya una práctica evaluativa fundamentada en un enfoque constructivista, y que parta del conocimiento real del alumnado (Greeno y Goldman, 1998). Un estado prolongado de exposición a pruebas de examen diseñadas sin considerar las ideas de anclaje de los aprendices y la estimulación del recuerdo llevaría a los sujetos menos aventajados, igual que a los más creativos, a un estado de negación y, probablemente, de abandono académico (Novak, 2010). Esto implica el problema de en qué orden o patrón se presenta el material evaluativo con la finalidad de alcanzar la mejor aproximación al conocimiento de la materia de estudio que el docente desea medir (Brunner, 2006).

Como mencionamos anteriormente, el aprendiz AICLE no solo ha de aprender el nuevo conocimiento curricular que se le presenta; igualmente ha de soportar el estrés que supone la evaluación en una lengua no materna (L2). Este alumnado también ha de afrontar los aspectos intrínsecos de la lengua vehicular sobre los cuales "Uno de los elementos más exigentes de la escolarización es la 'demanda' de la prueba de que los estudiantes han aprendido lo que se ha enseñado” (Mintzes, Wandersee, y Novak, 1998, p. 250). Así, opinamos que el proceso de enseñanzaaprendizaje-evaluación ha de ser considerado y diseñado en su conjunto con la finalidad de evitar la memorización y proveer al aprendiz bilingüe de estrategias metacognitivas, de comprensión y de representación de la información aprendida. Del mismo modo, y desde la perspectiva que nos mantiene vinculados a la enseñanza bilingüe, entendemos que las respuestas manifestadas por los examinandos en una escuela bilingüe tendrían que ser igualmente valoradas si se realizasen en ambas lenguas: L1 o L2. De esta forma se evitarían situaciones como las manifestadas por Perrenoud (1991), quien afirma que un elevado número de aprendices pasan por los sistemas educativos con la única aspiración de evitar el mayor número de fracasos académicos, en lugar de aspirar a adquirir el mayor conocimiento posible.

Otra práctica asociada al anterior proceso consistiría en la negociación del aprendizaje en un clima de aula que estimule la participación y el desarrollo normal del ego del aprendiz. Entendemos que, especialmente en las etapas iniciales del desarrollo cognitivo, el sentimiento de éxito o fracaso influiría en el subsecuente aprendizaje del alumnado en los programas bilingües. Las etapas más avanzadas del desarrollo normal del ego en un individuo dependen de otras etapas iniciales del desarrollo del yo (Ausubel, 1996). Por esta razón, la enseñanza básica en lengua extranjera ha de servirse de los recursos o instrumentos que permiten el aprendizaje significativo de los aprendices bilingües sin asaltar en modo alguno el ego de este alumnado. Probablemente debido a un material deficientemente elaborado, el ego de los aprendices se observa en ocasiones menoscabado en las escuelas (Novak, 2010). Evitar este tipo situaciones en las aulas es fundamental puesto que, como consecuencia de una malformación del ego, algunos individuos desarrollarían determinadas patologías y neurosis (Ausubel, 2002a). 


\section{PLANTEAMIENTO METODOLÓGICO}

La propuesta de innovación presentada tiene su origen en la formación específica del profesorado AICLE en la elaboración del material de evaluación potencialmente significativo para la mejora de los resultados académicos de los discentes inmersos en los programas bilingües que realizarán estas pruebas de examen. Para Ausubel (1952), la enseñanza implica una meticulosa consideración de la pedagogía diferencial para dar respuesta a los distintos tipos de aprendizaje que manifiestan los aprendices satelizadores (demandan la ayuda del docente) y no satelizadores (no demandan la ayuda del docente). En los programas bilingües (inglés-español) el docente ha de poseer determinadas estrategias que le permitan elaborar instrumentos de evaluación alejados de la literalidad en la respuesta y lo memorístico. No obstante, aunque el material de evaluación elaborado requiera respuestas abiertas y creativas existen casos en los que el alumnado prefiera memorizar el material de examen.

\subsection{Mapas conceptuales en la evaluación}

Parece bastante probable que el resultado académico más o menos exitoso de un discente de Primaria, así como el mayor o menor tiempo por el cual este retiene en su estructura cognitiva lo aprendido, esté influenciado por el modo en el cual el instructor diseña, implementa y evalúa los conocimientos (Hoffmann, 2003). Aunque el material de transferencia se elabore de manera potencialmente significativa y se cumplan todos los criterios fundamentales del aprendizaje significativo, si las pruebas de evaluación se diseñan para medir un aprendizaje memorístico, el resultado académico del aprendiz sería inferior (como ocurriría si el sujeto se siente obligado a representar respuestas cerradas y literales en las pruebas sumativas), puesto que tenderá a memorizar el material de estudio (Gómez, Lirio, Gómez-Barreto, Garrote, 2018). Así, un aprendiz familiarizado con modelos de examen cerrados que exijan respuestas literales tenderá a memorizar el material con la única finalidad de superar dicha prueba, independientemente de que el material presentado sea potencialmente significativo. La alternativa a este problema consistiría en la elaboración de modelos de examen que incorporen mapas conceptuales para comprobar lo aprendido por los sujetos (Ruiz-Primo, 2000).

\subsection{Literalidad vs. sustancialidad}

La demanda de respuestas literales o contrariamente de respuestas sustanciales o abiertas en una prueba de examen influenciaría de igual forma los resultados obtenidos por los estudiantes. Este factor se debería a la forma en la cual el docente solicitaría de los examinandos mayor o menor literalidad o sustancialidad de respuesta en la prueba. Es decir, en una prueba de examen de Natural Science el aprendiz encontraría más fácil definir determinados conceptos sustancialmente que de forma literal, sin que por ello afecte al significado de la definición. La definición de la esencia de un concepto (atributos de criterio) "no depende del uso exclusivo de unas palabras concretas y no otras; el mismo concepto o la misma proposición se podrían expresar mediante un lenguaje sinónimo y se transmitiría con precisión el mismo significado" (Ausubel, 2002b, p. 127). Considerando las ideas anteriores, podría decirse que de una prueba de examen potencialmente significativa se obtendrían respuestas sustanciales; mientras que de una prueba de examen basada en el aprendizaje memorístico se obtendrían respuestas literales o cerradas. Aunque en ambos casos la representación refleja el fondo sustancial de la información aprendida por los sujetos, las respuestas pertenecientes al primer supuesto serían sustanciales, abiertas, razonadas, deductivas e inferenciales; mientras el segundo supuesto es más proclive a la obtención de respuestas cerradas, literales, no razonadas e inductivas. 


\subsection{Elaboración jerárquica de las preguntas}

Otro aspecto que facilitaría el proceso de respuesta en un examen es la elaboración de modelos que incorporen una estructura jerárquica pertinente al nivel de exigencia y abstracción del conocimiento demandado por el examinador. Este modo de organización poseería su fundamento en la subordinación correlativa propuesta por Ausubel (2002b). Puesto que en el proceso de asimilación del material potencialmente significativo el cerebro tiende a asociar, organizar y almacenar las nuevas abstracciones de forma jerárquica y ordenada, opinamos que la medición de lo aprendido igualmente tendría que seguir el mismo patrón de generalidad y concreción. La idea de elaborar modelos de exámenes basados en este principio consiste en la estimulación del recuerdo y su empleo extensivo. Es decir, dependiendo de la cantidad y grado de abstracción de la información demandada, así será la transformación cognitiva que permita al aprendiz demostrar su conocimiento real. Por ejemplo, un mayor nivel de abstracción, por parte del alumnado, sobre la materia aprendida (subconceptos de un concepto) implicaría el acierto de un número mayor de respuestas. Del mismo modo el alumnado podría inferir determinadas respuestas conociendo cuáles son y qué significan los atributos de criterio de un concepto (Ausubel et al., 1983).

\section{EVALUACIÓN DE LA REFLEXIÓN}

La evaluación mediante mapas conceptuales se justifica porque el dominio de los conceptos y la construcción de los esquemas de aprendizaje han estado lejos de constituir el centro de la enseñanza (Niemelä, Mikolainen y Vourinen, 2018). Así, en este documento planteamos la importancia del uso de los mapas conceptuales como instrumento para la construcción consciente y deliberada del conocimiento del alumnado. Este crecimiento consciente contribuiría al mejor resultado de aprendizaje del alumnado, además de conformarse como una oportunidad para la formación del ego académico durante el proceso de evaluación de los aprendizajes. En este caso concreto hemos seleccionado una estrategia metacognitiva, puesto que el mapa conceptual se convierte en una representación visual de la comprensión del alumnado. Tales representaciones habrán sido previamente trabajadas de manera conjunta con los contenidos curriculares, los cuales se convierten en la esencia del proceso de enseñanza-aprendizaje existiendo así una coherencia entre la forma en que se ha desarrollado el contenido y los instrumentos empleados en la evaluación. Así, una sugerencia que planteamos a partir de la revisión teórica descrita consiste en el establecimiento de criterios u objetivos que ayuden al docente a diseñar y calificar la prueba escrita en la que se utilice los mapas conceptuales:

1. Formular preguntas de enfoque que sirvan de referencia y punto de partida para generar oportunidades de clarificación de ideas en los examinandos, ayudándoles en la estructuración de su pensamiento a partir del conocimiento adquirido y demandado en la prueba.

2. Guiar en la importancia de los organizadores visuales como base para dar respuestas bien definidas en la elaboración de los mapas conceptuales en los exámenes.

3. Utilizar los facilitadores visuales para establecer las relaciones y conexiones que permitan evidenciar el dominio del conocimiento del estudiante en la evaluación sumativa.

Los criterios anteriormente descritos están relacionados con resultados de investigaciones anteriores que afirman que la evaluación mediante mapas conceptuales se convierte en un instrumento útil para docentes y discentes (Williams, 1998). Del mismo modo, la evaluación mediante mapas conceptuales permitiría la retroalimentación 
percibida como un aspecto positivo por los aprendices (Gilbert, Justi y Ferreira, 2007; Niemelä et al, 2018), lo que de algún modo favorecería un mejor desarrollo académico del ego de los aprendices. En una investigación realizada por Niemelä et al. (2018), los propios aprendices mencionan que la elaboración de mapas conceptuales les parece una forma interesante y creativa de mostrar lo que saben. Los sujetos encuestados igualmente afirman que los mapas conceptuales les resultan unos instrumentos útiles para representar lo aprendido. Consecuentemente, invitamos a los docentes de Primaria a incorporar el uso de esta estrategia como forma de valorar el aprendizaje de los estudiantes, así como también favorecer los procesos cognitivos, las estrategias metacognitivas y emocionales en la construcción y valoración de aprendizajes significativos, profundos y duraderos.

\section{REFERENCIAS}

Ausubel, D. P. (1952). Ego Development and the Personality Disorders. New York, NY: Grune y Stratton.

Ausubel, D. P. (1996). Ego Development and Psychopathology. New Brunswick, NJ: Transaction Publishers.

Ausubel, D. P. (2002a). Theory and problems of adolescent development. New York, NY: Writers Club Press.

Ausubel, D. P. (2002b). Adquisición y retención del conocimiento. Barcelona: Paidós Ibérica.

Ausubel, D. P., Novak, J. D. y Hanesian, H. (1983). Psicología educativa: Un punto de vista cognoscitivo (2ª ed., reimpresión 2010). México: Trillas.

Black, P. y Wiliam, D. (1998). Assessment and Classroom Learning. Assessment in Education, 5(1), 7-78.

Bruner, J. (2006). In Search of Pedagogy (Vol. 1). New York, NY: Routledge.

Claxton, G. (1984). Live and Learn: An Introduction to the Psychology of Growth and Change in Everyday Life. London: Harper and Row.

Dobson, A., Pérez-Murillo, M. D. y Johnstone, R. (2010). Programa de educación bilingüe en España: Informe de evaluación. Madrid: MEC-British Council.

Edmondson, K. M. (2000). Assessing Science Understanding Through Concept Maps. En J. J. Mintzes, J. H. Wandersee y J. D. Novak (Eds.), Assessing Science Understanding: A Human Constructivist View (pp. 15-40). San Diego, CA: Academic Press.

Gilbert J., Justi, K. y Ferreira P. (2007). Modelling, Visualization, and the Development of an Understanding of the Levels of Representation in Chemical Education. En ESERA 2007 Conference, August 21-25 at Malmö University. Malmö (Sweden).

Gipps, C. (1992). Beyond Testing: Towards a Theory of Educational Assessment. Washington, DC: The Falmer Press.

Gómez, J., Lirio, J., Gómez-Barreto, I. y Garrote, D. (2018). Programas bilingües y Educación Primaria: mapas conceptuales para la mejora del aprendizaje en L2. En E. D. López-Meneses, D. Cobos-Sanchiz, A. H. MartínPadilla, L. Molina-García y A. Jaén-Martínez (Eds.), Experiencias Pedagógicas e Innovación Educativa: Aportaciones Desde la Praxis Docente e Investigadora: Vol. I. (pp. 24-37). Barcelona: Octaedro 
Greeno, J. y Goldman, S. (1998). Thinking practices in mathematics and science learning. New York, NY: Routledge.

Hoffmann, B. (2003). The Tyranny of Testing. New York, NY: Dover.

Joshi, R. M., Sangahvi, S. J., Upadhayaya, D. P., Chauhan, A. y Halvadia. S. (2012). Effect of Examination Stress on the Plasma Cortisol Level. National Journal of Medical Research, 2(4), 435-438.

Mintzes, J. J., Wandersee, J. H. Y Novak, J. D. (1998). Teaching science for understanding: A human constructivist view. San Diego, CA: Academic Press.

Ng, V., Koh, D. y Chia, S.-E. (2003). Examination Stress, Salivary Cortisol, and Academic Performance. Psychological Reports, 93(3), 1133-1134. Recuperado de https://doi.org/10.2466/pr0.2003.93.3f.1133

Niemelä, P., Mikkolainen, V. y Vuorinen, J. (2018). Compute mindlessly. Not! Map consciously. Universal Journal of Educational Research, 6(11), 2669-2678. Recuperado de https://doi.org/10.13189/ujer.2018.061133

Novak, J. D. (2010). Learning Creating and Using Knowledge: Concept Maps as Facilitative Tools in Schools and Corporations. New York, NY: Routledge.

NUT (2010, October 18). Make assessment measure up. Recuperado de https://www.atl.org.uk/policy-and-campaigns/policy-posts/make-assessment-measure-0

Perrenoud, P. (1991) Towards a Pragmatic Approach to Formative Evaluation. En P. Weston (Ed.), Assessment of Pupils'Achievement: Motivation and School Success, (2) (pp. 79-101). Amsterdam: Swets and Zeitlinger.

Ruiz-Primo, M. (2000). On the use of concept maps as an assessment tool in science: What we have learned so far. REDIE: Revista Electrónica de Investigación Educativa, 2(1). Recuperado de: https://redie.uabc.mx/redie/article/ view/16/29.

Shepard, L. A., y Smith, M. L. (1987). Effects of Kindergarten Retention at the end of First Grade. Psychology in the Schools, 24(4), 346-357.

Williams, C. G. (1998). Using Concept Maps to Assess Conceptual Knowledge of Function. Journal for Research in Mathematics Education, 29(4) 414-421.

\section{INFORMACIÓN SOBRE EL AUTOR}

José Luis Gómez Ramos es doctor en Humanidades, Artes, y Educación por la Universidad de Castilla-La Mancha, España. Asimismo es profesor, en la modalidad bilingüe, del área de Didáctica y Organización Escolar en el departamento de Pedagogía de la Facultad de Educación del Campus Albacete en la Universidad de CastillaLa Mancha (UCLM), España. Como actividad académica, dirige los Prácticum I y II del alumnado bilingüe. Del mismo modo, dirige los Trabajo Fin de Grado elaborados en inglés. Sus líneas de investigación se centran en la formación del profesorado bilingüe, así como en los procesos de enseñanza y aprendizaje en el aula bilingüe de educación primaria.

$\triangle$ joseluis.gomez@uclm.es 
Juan Lirio Castro. Doctor en Pedagogía por la Universidad Complutense de Madrid. Asimismo, es Profesor de Educación Social en la Universidad de Castilla-La Mancha. Actualmente lidera y dirige en la misma Universidad el Grupo de Investigación en Gerontología Social y Educativa (GESED). Las líneas de investigación del Dr. Castro se focalizan en los distintos procesos educativos, aunque especialmente en los procesos educativos vinculados a la educación social. Del mismo modo realiza investigaciones relacionadas con la educación de las personas mayores. En cuanto a la investigación internacional el Dr. Castro participa en distintos congresos cuyas líneas de investigación se focalizan en la educación y formación de las personas mayores.

\section{juan.Lirio@uclm.es}

Isabel María Gómez Barreto es Doctora en Ciencias de la Educación, Universidad Nacional de Educación a Distancia (UNED), España. Asimismo es Profesora del área de Didáctica y Organización Escolar en el departamento de Pedagogía de la Facultad de Educación del Campus Albacete en la Universidad de Castilla-La Mancha (UCLM), España. Como actividad docente dirige Trabajos de Fin de Grado y Máster desde hace 6 años, entre los cuales ha dirigido de forma virtual a dos de ellos pertenecientes a estudiantes Erasmus. La Dra. Barreto ha participado en diversos proyectos de innovación docente, entre los cuales se encuentran "Ansiedad de los estudiantes ante el Trabajo Fin de Grado" y "Pensamiento y aprendizaje: herramientas y estrategias didácticas para el profesorado". Su principal línea de investigación se focaliza en el pensamiento visible.

$\triangle$ isabelmaria.gomez@uclm.es

Rosa María Serna Rodríguez es doctora y licenciada en Filosofía y Ciencias de la Educación por la Facultad de Filosofía, Psicología y Ciencias de la Educación de la Universidad de Murcia. Asimismo es Licenciada en Antropología Social y Cultural por la Universidad Complutense de Madrid. Como actividad académica imparte asignaturas de investigación y evaluación en la intervención socioeducativa y métodos de investigación y diagnóstico en Educación. Del mismo modo, es profesora en el en el Máster Universitario en Profesor de Educación Secundaria Obligatoria y Bachillerato, Formación Profesional y Enseñanza de Idiomas. Su línea de investigación se focaliza en los aspectos jurídicos y gestión de recursos en materia de discapacidad. 